Rapid progress in the field of adult cells reprogramming back into a stem cell-like fate revealed shared mechanisms of action with tumoural reprogramming. A hallmark of stem cells - self-renewal and differentiation potential - seems to be tightly interlaced with large proliferation capacity and cellular plasticity of cancer cells. In this review, we briefly summarise the core transcription factors critical to maintenance of ES cell signature and overexpressed in many types of cancer, as well as signalling pathways involved in both induced pluripotency and oncogenesis, with particular regard to the role of tumour suppressor p53.

Key words: cancer, stem cells, reprogramming, induced pluripotent stem cells, p53.

Contemp Oncol (Pozn) 2015; 19 (1A): A16-A21 DOI: $10.5114 /$ wo. 2014.4713

\section{Oncogenesis and induced pluripotency - commonalities of signalling pathways}

\author{
Marta Klimczak $1,2,3$ \\ ${ }^{1}$ The International Institute of Molecular and Cell Biology, Warsaw, Poland \\ 2Postgraduate School of Molecular Medicine, Medical University of Warsaw, Poland \\ ${ }^{3}$ Gene Therapy Laboratory, Department of Cancer Immunology, Greater Poland Cancer \\ Centre, Poznan, Poland
}

\section{Introduction}

The multiple similarities between embryonic stem (ES) cells and cancer cells have led to widespread interest of researchers to discover molecular mechanisms that link these two cell types. Embryonic stem cells and cancer cells share certain common characteristics, such as the ability to self-renew and block in differentiation [1]. Interestingly, upon transplantation into immunodeficient mice, both ES cells and cancer cells form benign and malignant tumours, respectively. Moreover, both cell types have a rapid cell cycle and high telomerase activity, which result in uncontrolled proliferation [2]. The scientific breakthrough of Takahashi and Yamanaka in creating induced pluripotent stem (iPS) cells opened the door to broad stem cell research to understand the mechanisms involved in dedifferentiation. Importantly, the cocktail of four reprogramming factors is composed of two well-known proto-oncogenes, c-Myc and Klf4, which further connect induced pluripotency and oncogenesis. Here, we present a current understanding of transcriptional regulators that are critical to maintenance of self-renewal and are overexpressed in many human cancers. We also discuss the commonalities in signalling pathways and the role of tumour suppressor p53 in the regulation of reprogramming and tumorigenesis.

\section{Transcriptional regulation of dedifferentiation in tumorigenesis and induced pluripotency}

The connection between oncogenesis and induced pluripotency is commonly discussed by the fact that the core pluripotency genes involved in the reprogramming process also play a central role in tumourigenicity. The cocktail of Yamanaka factors [3] that enables the dedifferentiation of somatic cells to a stem cell-like fate is actually composed of well-known oncogenes, such as c-Myc and Klf4, or genes that exhibit high expression in various types of cancer, such as Oct3/4 and Sox2.

\section{Oct3/4}

Oct3/4, besides being a master player in induced pluripotency, plays a leading role in embryogenesis, and its correct level of expression is a critical requirement for initial formation of mammalian embryo. Not only knockdown, but also overexpression of Oct $3 / 4$ activates the differentiation to trophoectoderm and primitive endoderm or mesoderm, respectively [4]. The upregulation of Oct3/4 is characteristic of several types of human cancers, including pancreatic cancer, gastric cancer, oral squamous cell carcinoma, seminoma, and bladder cancer [5-7]. Furthermore, it has been demonstrated that Oct3/4 positively regulates the progression of carcinomas by stimulating dedifferentiation of cancer cells and acquiring cancer stem cell 
(CSC)-like phenotype, that in consequence leads to epithelial-mesenchymal transition (EMT), and development of tumour-initiation abilities, promoting metastases and formation of drug-resistant phenotype [8]. In agreement with this, Oct3/4 correlates with poor prognosis of human gliomas [9], melanoma [10], and lung adenocarcinoma [11].

\section{Sox2}

Another key factor in ES cell self-renewal, Sox2, is closely associated with many types of cancer. Overexpression of Sox2 imposes cancer stem cell-like properties, further influencing the tumour formation capacity and tumour aggressiveness in several types of cancer, such as squamous skin tumours [12], lung cancer [13, 14], and serous ovarian carcinoma [15]. Furthermore, Sox2 has been demonstrated to be involved in mechanisms of chemoresistance [13, $15,16]$. It was recently shown that a high level of Sox2 is associated with tamoxifen resistance, which develops by Sox2-dependent activation of Wnt signalling pathway in cancer stem/progenitor cells [16].

\section{KIf4}

Besides being part of the Yamanaka factors, Klf4 is also a bifunctional player in the process of carcinogenesis - it has been suggested that it acts both as an oncogene and a tumour suppressor depending on the different cellular context [17]. For example, Klf4 inhibits cancer cell invasion in primary lung cancer by suppressing the expression of SPARC, which is an extracellular matrix protein involved in tumour development and metastasis [18]. Tumour suppressing function of Klf4 in some types of cancer is also associated with its capacity to prevent epithelial-mesenchymal transition, a critical process in cancer progression and metastasis. It functions through repression of key mesenchymal markers, such as Slug and Snail in hepatocellular carcinoma and breast cancer, respectively [19, 20]. Klf4 maintains also the expression of E-cadherin, preventing EMT in mammary epithelial cells and supporting its metastasis suppressive role in breast cancer [21]. On the other hand, Klf4 was found to play a potent oncogenic role in colon cancer by enriching spheroid cells with cancer stem cell (CSC) markers and mesenchymal markers [22] Similarly, Klf4 is believed to act as an oncogene in mammary tumorigenesis by maintaining stem cell-like features that seem to be inconsistent with its tumour suppressing role described in ref 21.

\section{c-Myc}

c-Myc, one of the first proto-oncogenes discovered is activated in nearly $70 \%$ of all human cancers [23]. Undoubtedly, it plays a crucial role in tumorigenesis as well as being partially responsible for teratoma formation in iPS-derived grafts [24]. Being one of the Yamanaka reprogramming factors, c-Myc has given rise to a lot of controversy as it carries a risk of oncogenic transformation that excludes cells with exogenous expression of c-Myc from potential clinical application. Although it is possible to generate iPS cells without c-Myc, the process is highly inefficient [24]. Moreover, c-Myc has an essential positive effect upon iPS cell formation in terms of achieving mature iPS cells that are more prone to form chimeric mice [25]. Further studies have suggested that c-Myc is actually not a critical member of the core pluripotency network as its targets are involved more in cellular metabolism, cell cycle, and protein synthesis than in developmental and transcription-associated processes [26]. It has been proposed that the transcription regulatory mechanism that controls ES cell fate can be divided into three modules: core, Polycomb, and Myc [27]. These three regulatory units are functionally separable; therefore, the overall ES cell transcription network is composed of distinct units. In ES cells, the Polycomb module was inactive but the core and Myc modules were upregulated, whereas in cancer cells the Myc module was active but the core unit that underlies the ES cell fate was not overexpressed [1, 27]. These findings suggest that similarities in gene expression signature of ES cells and cancer cells can be a result of the prevalent contribution of Myc regulatory network rather than that of an ES-cell specific network [1].

\section{Signalling pathways in cancerogenesis and acquisition of pluripotency}

\section{Wnt/ $\beta$-catenin signalling}

The Wnt signalling pathway is implicated in a wide range of cell behaviours that occur throughout development. It plays a vital role in cell proliferation, cell movement, establishment of tissue polarity, cell fate decisions, as well as in stem cell maintenance [28]. The Wnt pathway has two distinct signalling branches: the canonical Wnt pathway that is characterised by stabilisation and accumulation of $\beta$-catenin, which then translocates to the nucleus and participates in a transcriptome regulation; and the noncanonical pathway that is $\beta$-catenin-independent $[28,29]$. The canonical branch of the Wnt pathway has been shown to share molecular targets involved in regulation of self-renewal in the context of both stem cells and cancer [29, 30]. The unquestionable role of Wnt signalling in embryogenesis has been supported by its contribution in the maintenance of pluripotency in mouse and human ESCs [31-33] as well as in the self-renewal of adult stem cells in multiple types of tissues [34]. Wnt signal enhances the effect of LIF in maintenance of the self-renewal of mouse ES cells [32] and upregulates Stat3 thus helping to inhibit the ES cell differentiation even in the absence of LIF [35]. Furthermore, studies of a terminal component of canonical Wnt pathway, Tcf3, revealed that this factor co-occupies promoter regions throughout the genome associated with ESC-specific transcription factors such as Oct3/4 and Nanog [36]. Paracrine and autocrine Wnt signals also inhibit the differentiation to EpiSCs, so they prevent the transition from naïve to primed pluripotency [37]. Additionally, $\beta$-catenin regulates the expression of the telomerase subunit TERT through interaction with Klf4 and Wnt target gene c-Myc, uncovering the next link between stem cells and oncogenic potential [38]. Taking into consideration the fact that signalling pathways involved in ES cell self-renewal and those that contribute to somatic cell reprogramming often overlap, the role of Wnt pathway 
in induced pluripotency was expected to be predictable. However, there are still some contradictions that make it unclear [39]. It has been shown that $\beta$-catenin might promote reprogramming of fibroblasts to pluripotency [3]. These data were supported by the fact that reprogramming can be triggered by accumulation of $\beta$-catenin in a dose-dependent manner, and activation of Wnt pathway noticeably enhances the ability of ES cells to reprogram somatic cells by fusion [40]. Further studies shed light on the biphasic effect of Wnt pathway in reprogramming. It has been suggested that Wnt signalling has an inhibitory role in the early stage of dedifferentiation but stimulates the process of induced pluripotency in its late stage. These changes are also accompanied by the requirement of different transcriptional effectors from among Tcf1, Lef1, Tcf3, and Tcf4 [41]. Very recent work has confirmed the fact that $\beta$-catenin is crucial for reprogramming because it stimulates the endogenous pluripotent gene expression. However, this effect is transient and $\beta$-catenin activation occurs only at the initial stage of reprogramming and is no longer required for pluripotent stem cell maintenance [42].

\section{Hedgehog signalling}

The Hedgehog $(\mathrm{Hh})$ pathway is a major mediator of many fundamental processes regulating development, mainly through the control of cell fate, proliferation, survival, and differentiation [43, 44]. Aberrant Hh pathway activity is associated with aetiology of basal cell carcinoma and a subset of medulloblastoma [45]. A variety of other human cancers such as pancreas, lung, prostate, breast, brain, and gastrointestinal cancers could also be dependent on this pathway [44, 45]. Hedgehog signalling is often involved in the stimulation of cancer stem cell growth and promotion of an embryonic stem cell-like gene expression signature [46]. Therefore, it has a critical impact on tumour growth, survival, recurrence, and metastasis in many types of human cancer [47]. It has been discovered that activity of Gli, a central player in Hh pathway, is enhanced by loss of tumour suppressors, including p53 and PTEN, and by several oncogenic proteins, such as Ras, Myc, and Akt $[47,48]$. Gli also upregulates the expression of Nanog by binding to its cis-regulatory sequences. Additionally, loss of p53, a key event promoting cell stemness and oncogenesis, leads to the activation of Hh signalling and consequently contributes to further Nanog upregulation [49]. Activators of $\mathrm{Hh}$ pathway in combination with Oct3/4 have been described to be capable of iPS generation from mouse embryonic and adult fibroblasts, indicating that they can replace Sox2, c-Myc, and Klf4 in reprogramming process [50].

\section{Notch signalling}

The Notch signalling pathway is evolutionarily conserved and plays a critical pleiotropic role in the proliferation, self-renewal, and differentiation of many distinct progenitor cell and stem cell types [51]. It acts differentially in a tissue- and dose-dependent manner. As an example, Notch can both promote and suppress the proliferation of mammary gland epithelial cells depending on its activity level. Additionally, Notch activation can promote both tumour suppression and oncogenesis, depending on the cell lineage, even within the same tissue. Such completely opposite biological effects have been described in several solid tumours including breast, prostate, lung, and oral squamous cell carcinoma [52]. Recent studies revealed that Notch signalling is an essential regulator of pluripotency and self-renewal of iPS cells. It activates ROCK implicated in keratinocyte differentiation leading to impaired self-renewal of human induced pluripotent stem cells and cellular differentiation [53]. Furthermore, signalling through Notch pathway is a major obstacle in an early stage of dedifferentiation of both mouse and human keratinocytes into iPS cells. Finally, inhibition of Notch enables production of human iPS cells without KIf4 and c-Myc by suppressing p21 in a p53-independent manner [51].

\section{Dysregulation of p53 pathway in cancer formation and reprogramming}

The p53 transcription factor, encoded by the human gene TP53, is a key tumour suppressor and regulator of genetic stability. It plays a critical role in cell cycle arrest, DNA repair, programmed cell death, senescence, and differentiation. The activation or repression of differentiation by p53 is dependent on the cell type and cell fate and acts through control of cell cycle and apoptosis as well as through direct transcriptional regulation [54]. A poorly differentiated phenotype has been shown to be associated with the most aggressive human tumours and occurs frequently as a result of ES-like signature [2]. Reprogramming induced by a set of Yamanaka factors also represents a clear example of dedifferentiation process starting from terminally differentiated cells such as fibroblasts and finally reaching the pluripotent state [54]. It has been reported that p53 serves as a potent barrier to somatic cell reprogramming and dramatically reduces the efficiency of dedifferentiation. Therefore, to improve the reprogramming capacity, many groups have been evaluating the effect of p53 signalling disruption by deleting or silencing the expression of genes involved in this pathway or expressing a mutated version of p53 $[55,56]$. Lower p53 level profoundly facilitates the reprogramming process, but it also leads to generation of iPS cells with chromosomal abnormalities and genome instability [56]. Additionally, mutant-p53-expressing cells exhibit higher potential to initiate malignant tumour formation than cells expressing wild-type p53 [57]. Therefore, there seems to be a striking commonality between improved reprogramming process and increased predisposition to cancer formation in the absence of p53. Here, we examine recent findings to evaluate the mechanisms of action of p53 involved in maintaining genomic integrity and regulating mesenchymal-to-epithelial transition, which are critical in both tumorigenesis and reprogramming.

\section{p53-mediated DNA damage response}

Genomic instability is a major characteristic of most cancers, which probably develops as a combined effect of DNA damage and failure in DNA damage response (DDR) 
[58]. In response to various abnormalities, such as telomere attrition, high level of reactive oxygen species (ROS), or oncogene-induced unscheduled replication, DNA damage response is activated. It is coordinated by cell-cycle checkpoint kinases, including ATM (ataxia telangiectasia mutated) and ATR (ATM and Rad3-related), which orchestrate p53-dependent repair pathways. Germline and somatic mutations in components of this checkpoint machinery constrain DNA damage repair enabling cells with genomic defects to proceed through the cell cycle intact, increasing the risk of mutagenesis and predisposing to cancer formation [58].

A p53-dependent DNA damage response is also a key modulator of reprogramming as it prevents the acquisition of the pluripotent state of cells with various types of DNA damage, such as DNA repair defects, dysfunctional telomeres, or damage caused by exogenous agents. In cells with alterations in DNA structure, the process of dedifferentiation is usually terminated in its initial stage by p53-mediated activation of senescence genes, such as Cdkn1a, and also by inhibition of ES cell-enriched genes, such as Oct3/4, Sox2, Myc, and Esrrb, and activation of differentiation-associated genes [59].

\section{The role of $\mathrm{p} 53$ in regulation} of epithelial-mesenchymal transition

Changes between epithelial and mesenchymal states, defined as epithelial-mesenchymal transition (EMT) and mesenchymal-epithelial transition (MET), significantly regulate cellular plasticity. They are hallmarks of embryonic development and tumour progression. The term EMT refers to a process by which polarised cells lose their ability of cell-cell adhesion and gain the mesenchymal phenotype characterised by mobility, increased resistance to apoptosis, and the ability to leave the primary tumour site and migrate to distant sites. Therefore, both EMT and MET have pivotal roles in tumorigenesis: EMT contributes to invasion, tumour dissemination, and development of resistance to commonly used therapies; $M E T$, in a reverse process to EMT, is associated with formation of distant metastases [60].

Interestingly, recent work has suggested that p53 restricts MET by inhibition of Klf4-dependent activation of epithelial transcriptional genes [54]. In addition, the repressive effect on the transcription of epithelial genes has been observed as early as one day after infection of cells with ectopic reprogramming factors, indicating that p53-mediated inhibitory effect on MET is not a result of its ability to modulate proliferation, senescence, cell cycle arrest, or apoptosis. These data also provided a mechanistic insight into the oncosuppressive role of p53 as it influences the genome-wide inhibition of Klf4-transcriptional program and can even restrict the oncogenic potential of Klf4.

Additionally, it has been suggested that p53 modulates changes between epithelial and mesenchymal states by regulation of miRNA. For instance, p53-dependent activation of miRNA-34 serves to antagonize Snail1, a transcriptional repressor which is linked to cancer cell EMT process, as well as to inhibit Snail1 regulatory molecules such as $\beta$-catenin,
LEF1, and Axin2, which are commonly known to be involved in Wnt signalling pathway [61]. Interestingly, miRNA-34 and p21 are cooperative regulators of somatic reprogramming and act downstream of p53 [62]. It has been demonstrated that enhanced activity of miR-34 leads to repression of reprogramming factors including Nanog, Sox2, and $\mathrm{n}-\mathrm{Myc}$, consequently inhibiting the process of acquisition of pluripotency. p53 also plays a role in regulation of EMT through transcriptional activation of other miRNA, such as miRNA-200c, which indirectly modulates the expression of E-cadherin, a known epithelial cell marker [63]. miRNA-200c was shown to inhibit ZEB1/2, which is a transcriptional repressor of E-cadherin; therefore, it acts as a p53-dependent factor involved in maintaining epithelial character and preventing development of stem cell properties.

\section{Conclusions}

In this review we discussed the latest findings in the area of biological parallelisms between induced pluripotency and oncogenesis. Through the large effort of researchers around the world, the molecular mechanisms of cellular stemness have been evaluated and light has been shed on the understanding of tumour formation as a process of dedifferentiation and acquisition of stem cell-like features. This concept has provided the starting point of new therapeutic approaches in which disruption of common signalling pathways involved in both normal and pathological dedifferentiation could have potential value in the treatment of cancer. However, there are still some unresolved aspects, like differences between normal and tumoural reprogramming, which have to be precisely understood in order to develop rational and effective therapies.

The author declares no conflict of interest.

The author would like to thank Wtodzimierz Krzyżosiak and Maciej Wiznerowicz for reading the manuscript prior to submission.

MK was supported by the National Science Centre grants No: 2012/06/A/NZ1/00089 and 6129/B/P01/2011/40.

\section{References}

1. Kim J, Orkin SH. Embryonic stem cell-specific signatures in cancer: insights into genomic regulatory networks and implications for medicine. Genome Med 2011; 3: 75.

2. Ben-Porath I, Thomson MW, Carey VJ, Ge R, Bell GW, Regev A, Weinberg RA. An embryonic stem cell-like gene expression signature in poorly differentiated aggressive human tumors. Nat Genet 2008; 40: 499-507.

3. Takahashi K, Yamanaka S. Induction of pluripotent stem cells from mouse embryonic and adult fibroblast cultures by defined factors. Cell 2006; 126: 663-76.

4. Niwa H, Miyazaki J, Smith AG. Quantitative expression of Oct-3/4 defines differentiation, dedifferentiation or self-renewal of ES cells. Nat Genet 2000; 24: 372-6.

5. Wasik AM, Grabarek J, Pantovic A, et al. Reprogramming and carcinogenesis - parallels and distinctions. Int Rev Cell Mol Biol 2014; 308: 167-203.

6. Koide H. Embryonic stem cells and oncogenes. In: Pluripotent Stem Cell Biology - Advances in Mechanisms, Methods and Models. InTech 2014; 41-61. 
7. Lu Y, Zhu H, Shan H, et al. Knockdown of Oct4 and Nanog expres sion inhibits the stemness of pancreatic cancer cells. Cancer Lett 2013; 340: 113-23.

8. Linn DE, Yang X, Sun F, et al. A Role for OCT4 in Tumor Initiation of Drug-Resistant Prostate Cancer Cells. Genes Cancer 2010; 1: 908-16.

9. Guo Y, Liu S, Wang P, et al. Expression profile of embryonic stem cell-associated genes Oct4, Sox2 and Nanog in human gliomas. Histopathology 2011; 59: 763-75.

10. Kumar SM, Liu S, Lu H, et al. Acquired cancer stem cell phenotypes through Oct4-mediated dedifferentiation. Oncogene 2012; 31: 4898-911.

11. Chiou S-H, Wang M-L, Chou Y-T, et al. Coexpression of Oct4 and Nanog enhances malignancy in lung adenocarcinoma by induc ing cancer stem cell-like properties and epithelial-mesenchymal transdifferentiation. Cancer Res 2010; 70: 10433-44.

12. Boumahdi S, Driessens G, Lapouge G, et al. SOX2 controls tumou initiation and cancer stem-cell functions in squamous-cell carcinoma. Nature 2014; 511: 246-50.

13. Chou Y-T, Lee C-C, Hsiao S-H, et al. The emerging role of SOX2 in cell proliferation and survival and its crosstalk with oncogenic signaling in lung cancer. Stem Cells 2013; 31: 2607-19.

14. Chen S, Xu Y, Chen Y, et al. SOX2 gene regulates the transcrip tional network of oncogenes and affects tumorigenesis of human lung cancer cells. PLoS One 2012; 7: e36326.

15. Bareiss PM, Paczulla A, Wang H, et al. SOX2 expression associates with stem cell state in human ovarian carcinoma. Cancer Res 2013; 73: 5544-55.

16. Piva M, Domenici G, Iriondo O, et al. Sox2 promotes tamoxifen resistance in breast cancer cells. EMBO Mol Med 2014; 6: 66-79.

17. Rowland BD, Bernards R, Peeper DS. The KLF4 tumour suppresso is a transcriptional repressor of p53 that acts as a context-dependent oncogene. Nat Cell Biol 2005; 7: 1074-82.

18. Zhou Y, Hofstetter WL, He Y, et al. KLF4 inhibition of lung cancer cell invasion by suppression of SPARC expression. Cancer Biol Ther 2010; 9: 507-13.

19. Lin Z-S, Chu H-C, Yen Y-C, Lewis BC, Chen Y-W. Krüppel-like factor 4 a tumor suppressor in hepatocellular carcinoma cells reverts epithelial mesenchymal transition by suppressing slug expression. PLoS One 2012; 7: e43593.

20. Yori JL, Seachrist DD, Johnson E, Lozada KL, Abdul-Karim FW, Chodosh LA, Schiemann WP, Keri RA. Krüppel-like factor 4 inhibits tumorigenic progression and metastasis in a mouse model of breast cancer. Neoplasia 2011; 13: 601-10.

21. Yori JL, Johnson E, Zhou G, Jain MK, Keri RA. Kruppel-like factor 4 inhibits epithelial-to-mesenchymal transition through regulation of E-cadherin gene expression. J Biol Chem 2010; 285: 16854-63.

22. Leng Z, Tao K, Xia Q, et al. Krüppel-like factor 4 acts as an onco gene in colon cancer stem cell-enriched spheroid cells. PLoS One 2013; 8: e56082.

23. Nilsson JA, Cleveland JL. Myc pathways provoking cell suicide and cancer. Oncogene 2003; 22: 9007-21.

24. Nakagawa M, Koyanagi M, Tanabe K, et al. Generation of induced pluripotent stem cells without Myc from mouse and human fibroblasts. Nat Biotechnol 2008; 26: 101-6.

25. Agency T. Crucial role of c-Myc in the generation of induced plurip otent stem cells. Stem Cells 2011; 29: 1362-70.

26. Kim J, Chu J, Shen X, Wang J, Orkin SH. An extended transcriptional network for pluripotency of embryonic stem cells. Cell 2008; 132 1049-61.

27. Kim J, Woo AJ, Chu J, Snow JW, Fujiwara Y, Kim CG, Cantor AB, Orkin SH. A Myc network accounts for similarities between embryonic stem and cancer cell transcription programs. Cell 2010; 143: 313-24.

28. Van Amerongen R, Nusse R. Towards an integrated view of Wnt signaling in development. Development 2009; 136: 3205-14.

29. Takebe N, Ivy SP. Controversies in cancer stem cells: targeting embryonic signaling pathways. Clin Cancer Res 2010; 16: 3106-12.

30. Dreesen O, Brivanlou AH. Signaling pathways in cancer and embryonic stem cells. Stem Cell Rev 2007; 3: 7-17.
31. Sato N, Meijer L, Skaltsounis L, Greengard P, Brivanlou AH. Main tenance of pluripotency in human and mouse embryonic stem cells through activation of Wnt signaling by a pharmacological GSK-3-specific inhibitor. Nat Med 2004; 10: 55-63.

32. Ogawa K, Nishinakamura R, Iwamatsu Y, Shimosato D, Niwa H. Synergistic action of Wnt and LIF in maintaining pluripotency of mouse ES cells. Biochem Biophys Res Commun 2006; 343: 159-66.

33. Cai L, Ye Z, Zhou BY, Mali P, Zhou C, Cheng L. Promoting human embryonic stem cell renewal or differentiation by modulating Wnt signal and culture conditions. Cell Res 2007; 17: 62-72.

34. Reya T, Clevers H. Wnt signalling in stem cells and cancer. Nature 2005; 434: 843-50.

35. Hao J, Li T-G, Qi X, Zhao D-F, Zhao G-Q. WNT/beta-catenin pathway up-regulates Stat3 and converges on LIF to prevent differentiation of mouse embryonic stem cells. Dev Biol 2006; 290: 81-91.

36. Cole MF, Johnstone SE, Newman JJ, Kagey MH, Young RA. Tcf3 is an integral component of the core regulatory circuitry of embryonic stem cells. Genes Dev 2008; 22: 746-55.

37. Ten Berge D, Kurek D, Blauwkamp T, Koole W, Maas A, Eroglu E, Siu RK, Nusse R. Embryonic stem cells require Wnt proteins to prevent differentiation to epiblast stem cells. Nat Cell Biol 2011; 13: $1070-5$

38. Hoffmeyer K, Raggioli A, Rudloff S, et al. Wnt/ $\beta$-catenin signaling regulates telomerase in stem cells and cancer cells. Science 2012; 336: 1549-54.

39. Miki T, Yasuda S, Kahn M. Wnt/ $\beta$-catenin signaling in embryonic stem cell self-renewal and somatic cell reprogramming. Stem Cell Rev 2011; 7: 836-46.

40. Lluis F, Pedone E, Pepe S, Cosma MP. Periodic activation of Wnt/ beta-catenin signaling enhances somatic cell reprogramming mediated by cell fusion. Cell Stem Cell 2008; 3: 493-507.

41. Ho R, Papp B, Hoffman JA, Merrill BJ, Plath K. Stage-specific regulation of reprogramming to induced pluripotent stem cells by Wnt signaling and T cell factor proteins. Cell Rep 2013; 3: 2113-26.

42. Zhang P, Chang W-H, Fong B, Gao F, Liu C, Al Alam D, Bellusci S, Lu W. Regulation of induced pluripotent stem (iPS) cell induction by Wnt/ $\beta$-catenin signaling. J Biol Chem 2014; 289: 9221-32.

43. Coni S, Infante P, Gulino A. Control of stem cells and cancer stem cells by Hedgehog signaling: pharmacologic clues from pathway dissection. Biochem Pharmacol 2013; 85: 623-8.

44. Gupta S, Takebe N, Lorusso P. Targeting the Hedgehog pathway in cancer. Ther Adv Med Oncol 2010; 2: 237-50.

45. Teglund S, Toftgård R. Hedgehog beyond medulloblastoma and basal cell carcinoma. Biochim Biophys Acta 2010; 1805: 181-208.

46. Tang S-N, Fu J, Nall D, Rodova M, Shankar S, Srivastava RK. Inhibition of sonic hedgehog pathway and pluripotency maintaining factors regulate human pancreatic cancer stem cell characteristics. Int J Cancer 2012; 131: 30-40.

47. Ruiz i Altaba A. Hedgehog signaling and the Gli code in stem cells, cancer, and metastases. Sci Signal 2011; 4: pt9.

48. Zbinden M, Duquet A, Lorente-Trigos A, Ngwabyt S-N, Borges I, Ruiz i Altaba A. NANOG regulates glioma stem cells and is essen tial in vivo acting in a cross-functional network with GLI1 and p53. EMBO J 2010; 29: 2659-74

49. Po A, Ferretti E, Miele E, et al. Hedgehog controls neural stem cells through p53-independent regulation of Nanog. EMBO J 2010; 29: 2646-58.

50. Moon J-H, Heo JS, Kim JS, et al. Reprogramming fibroblasts into in duced pluripotent stem cells with Bmi1. Cell Res 2011; 21: 1305-15.

51. Ichida JK, T C W J, Williams LA, et al. Notch inhibition allows oncogene-independent generation of iPS cells. Nat Chem Biol 2014; 10 632-9.

52. Ranganathan P, Weaver KL, Capobianco AJ. Notch signalling in solid tumours: a little bit of everything but not all the time. Nat Rev Cancer 2011; 11: 338-51.

53. Yugawa T, Nishino K, Ohno S-I, Nakahara T, Fujita M, Goshima N, Umezawa A, Kiyono T. Noncanonical NOTCH signaling limits self-renewal of human epithelial and induced pluripotent stem cells through ROCK activation. Mol Cell Biol 2013; 33: 4434-47.

54. Brosh R, Assia-Alroy Y, Molchadsky A, et al. p53 counteracts reprogramming by inhibiting mesenchymal-to-epithelial transition. Cell Death Differ 2013; 20: 312-20. 
55. Kawamura T, Suzuki J, Wang YV, Menendez S, Morera LB, Raya A, Wahl GM, Izpisúa Belmonte JC. Linking the p53 tumour suppressor pathway to somatic cell reprogramming. Nature 2009; 460: 1140-4.

56. Marión RM, Strati K, Li H, et al. A p53-mediated DNA damage response limits reprogramming to ensure iPS cell genomic integrity. Nature 2009; 460: 1149-53

57. Sarig R, Rivlin N, Brosh R, et al. Mutant p53 facilitates somatic cell reprogramming and augments the malignant potential of reprogrammed cells. J Exp Med 2010; 207: 2127-40.

58. Lord CJ, Ashworth A. The DNA damage response and cancer therapy. Nature 2012; 481: 287-94.

59. Li M, He Y, Dubois W, Wu X, Shi J, Huang J. Distinct regulatory mechanisms and functions for p53-activated and p53-repressed DNA damage response genes in embryonic stem cells. Mol Cell 2012; 46: 30-42.

60. Polyak K, Weinberg RA. Transitions between epithelial and mesenchymal states: acquisition of malignant and stem cell traits. Nat Rev Cancer 2009; 9: 265-73.

61. Kim NH, Kim HS, Li X-Y, et al. A p53/miRNA-34 axis regulates Snail1-dependent cancer cell epithelial-mesenchymal transition. J Cell Biol 2011; 195: 417-33.

62. Choi YJ, Lin C-P, Ho JJ, et al. miR-34 miRNAs provide a barrier for somatic cell reprogramming. Nat Cell Biol 2011; 13: 1353-60.

63. Chang C-J, Chao C-H, Xia W, et al. p53 regulates epithelial-mesenchymal transition and stem cell properties through modulating miRNAs. Nat Cell Biol 2011; 13: 317-23.

\section{Address for correspondence}

\section{Marta Klimczak}

Laboratory of Gene Therapy

Department of Cancer Immunology

Greater Poland Cancer Centre

Garbary 15

61-866 Poznan, Poland

e-mail: m.klimczak01@gmail.com 